\title{
RETENCIÓN DE ESTUDIANTES VULNERABLES \\ EN LA EDUCACIÓN UNIVERSITARIA CHILENA
}

\section{Antecedentes}

Chile ha transitado desde un sistema de educación superior elitista, financiado mayoritariamente por el Estado, a uno masivo y diversificado que se financia mayoritariamente con recursos privados. En la actualidad, la educación superior chilena es provista por 16 universidades estatales, 45 privadas, 43 institutos profesionales privados, 105 centros de formación técnica privados y 19 entidades de las fuerzas armadas y de orden y seguridad. En total, el sistema cuenta con más de 200 instituciones y 400 sedes educativas (CSE, 2008).

Este proceso de expansión ha sido impulsado principalmente por el crecimiento de la oferta y matrícula universitaria privada, que representa cerca del 88\% de la matrícula total de pregrado (CSE, 2008; OCDE, 2009). Las cifras indican que en 1990 el sistema terciario contaba con cerca de doscientos mil estudiantes de pregrado (Uribe, 2004). Diecisiete años más tarde (2007), la matrícula de pregrado ha alcanzado a 752.182 estudiantes.

Una de las razones del incremento es la fuerte expansión de expectativas educacionales de las familias. Cifras aportadas por la Encuesta Nacional de Actores del $\mathrm{CIDE}^{2}$ dan cuenta de esta expansión de aspiraciones en todos los estratos sociales de la sociedad chilena (CIDE, 2001, 2003).

La creciente aspiración por ingresar al sistema de educación superior se explica, en parte, por los altos retornos de la educación terciaria, especialmente la de tipo universitaria. En Chile, los retornos a la educación universitaria se estiman cercanos al veinte por ciento, cifra bastante superior a las retribuciones obtenidas por profesionales

2 Centro de Investigación y Desarrollo de la Educación, Universidad Alberto Hurtado. 
equivalentes en países de la $\mathrm{OECD}^{3}$, que alcanzan retornos promedio de 12\% (Psacharopoulos \& Patrinos, 2002, 2004). Por tanto, el acceso a la educación superior representa una posibilidad para mejorar el bienestar social de los estudiantes y sus familias.

La expansión de la matrícula y oferta de educación superior ha posibilitado incrementar la presencia de todos los segmentos sociales en el sistema, incluida la de los segmentos socioeconómicamente más carenciados. De acuerdo con cifras provistas por la Encuesta CASEN, entre 1990 y 2006 la participación en la educación superior de los hogares de menores ingresos (quintiles I, II, III) se ha triplicado, pasando de cuatro a catorce por ciento en el primer quintil; de seis a diecisiete por ciento en el segundo, y de diez a veinticuatro por ciento en el tercer quintil.

Antecedentes provistos por el Instituto Nacional de Estadísticas (INE) (2003) indican que cerca de un 70\% de los estudiantes que actualmente están ingresando al sistema de educación superior son primera generación en cursar estudios de nivel terciario. Para este grupo, el ingreso al sistema constituye un proceso de adaptación y aprendizaje de nuevos códigos prácticos y simbólicos, situación que los pone en desventaja respecto de otros estudiantes que cuentan con mayor capital cultural y que, por tanto, se encuentran más familiarizados con las demandas del sistema.

A pesar de su creciente participación, los estudiantes del nivel socioeconómico más bajo no se distribuyen de manera homogénea dentro del sistema de educación terciaria. Sólo un tercio de los alumnos pertenecientes a los tres primeros quintiles se encuentra estudiando en universidades, mientras que los dos tercios restantes se ubican en centros de formación técnica (PNUD, 2005). Esta distribución refleja la segmentación del sistema educativo chileno, que hace que los estudiantes de nivel socioeconómico bajo estén subrepresentados en el sistema superior universitario (OCDE, 2009).

Los datos de la encuesta CASEN sugieren que los estudiantes de los dos quintiles más ricos representan el $66 \%$ de la matrícula

3 Organisation for Economic Co-operation and Development. 
52 RETENCIÓN DE ESTUDIANTES UNIVERSITARIOS VULNERABLES - Andrea Canales, Danae de los Ríos

universitaria. Al analizar esta distribución entre universidades del Consejo de Rectores (CRUCH) y universidades privadas, las cifras indican que, en las primeras, los estudiantes de los quintiles más acomodados (IV y V) representan el 53\% de los matriculados, mientras que para las segundas los mismos estudiantes representan el 70\% de la matrícula (OCDE, 2009).

Por el contrario, los estudiantes de los dos quintiles más pobres (I y II) representan el $27 \%$ de la matrícula en las universidades del CRUCH y un $16 \%$ de la matrícula en universidades privadas (Donoso y Cancino, 2007). En consecuencia, las universidades del CRUCH contribuyen más significativamente a la incorporación de segmentos vulnerables que tienen una menor presencia y participación dentro del sistema de educación terciario (OCDE, 2009).

La mayor cobertura del sistema educativo chileno no se ha traducido en un aumento significativo en los niveles de titulación, pues la proporción de individuos que finaliza la educación superior sigue siendo relativamente baja. En 2005, si bien la matrícula bruta alcanzaba al 42\%, sólo un 13\% de la población entre 25 y 64 años contaba con estudios superiores completos (World Bank, 2005).

Por su parte, las cifras sobre retención en el sistema indican que un significativo número de estudiantes abandona su carrera en primer o segundo año, disminuyendo la proporción de estudiantes que desertan a partir de tercero (Himmel, 2002; CSE, 2008). La información disponible indica también que uno de cinco estudiantes abandona la carrera antes del primer año y que en segundo año sólo dos de tres permanece estudiando la carrera a la cual ingresaron inicialmente (CSE, 2007).

Antecedentes provistos por la OCDE (2009) sugieren que las tasas de éxito educativo varían según puntaje en las pruebas de admisión y tipo de establecimiento educativo, variables fuertemente asociadas al estrato o nivel socioeconómico de los alumnos. Tal como lo indica la OCDE (2009), los problemas de deserción pueden explicarse en parte por la inequidad del sistema secundario, que proporciona una formación deficiente a los estudiantes de menores 
ingresos y por la escasa capacidad de suplir dichas falencias en el nivel terciario.

De forma complementaria, trabajos recientes sobre deserción universitaria sugieren que los estudiantes chilenos que abandonan el sistema terciario lo hacen motivados por causas económicas, socioculturales y motivacionales. En relación con factores motivacionales y socioculturales, el estudio de Canales y De los Ríos (2007) sugiere que las expectativas de los estudiantes no están suficientemente asentadas, lo que genera baja motivación y satisfacción con las carreras escogidas.

Adicionalmente, una proporción de los estudiantes no tiene hábitos académicos fuertes al momento ingresar a la universidad, lo que produce un desacoplamiento de expectativas y demandas en la transición educación secundaria-universidad (Canales y De los Ríos, 2007). Este desajuste de expectativas y demandas provoca un comportamiento errático de los estudiantes que experimentan entradas y salidas intermitentes en el sistema terciario.

Los antecedentes sobre deserción sugieren que los estudiantes de nivel socioeconómico bajo no cuentan con soportes familiares, sociales e institucionales para atenuar los efectos de hitos problemáticos, tales como la pérdida de trabajo del jefe de hogar, nacimiento de hijos, enfermedades y muerte de familiares relevantes. La falta de estos soportes genera, en muchos casos, el abandono permanente del sistema educativo con graves costos de bienestar.

El contexto anteriormente descrito sugiere que los estudiantes de mayor vulnerabilidad social enfrentan importantes obstáculos una vez que ingresan a la educación superior. Dado que la presencia de los alumnos con estas características tenderá a crecer durante las próximas décadas, resulta de suma importancia comprender los factores que explican su potencial permanencia en el sistema terciario, de modo tal de facilitar su proceso formativo.

Este estudio pretende contribuir a la construcción de una visión más amplia de los problemas que enfrentan los estudiantes 
54 RETENCIÓN DE ESTUDIANTES UNIVERSITARIOS VULNERABLES - Andrea Canales, Danae de los Ríos

universitarios vulnerables, evitando la estigmatización o idealización de los mismos. Entender las condiciones que facilitan y dificultan su permanencia en el sistema universitario podría ser de gran utilidad para las instituciones de educación superior y los diseñadores de políticas públicas, que buscan desarrollar iniciativas adecuadas en este campo.

A continuación se proporcionan antecedentes para entender la permanencia de estudiantes vulnerables en el nivel terciario, antecedentes que aportan algunas ideas para comprender las barreras y dificultades enfrentadas, así como también los soportes y estrategias que facilitan su progresión dentro del nivel superior.

\section{Breve discusión bibliográfica}

Existen numerosos estudios sobre la permanencia de estudiantes vulnerables en la educación superior. Gran parte de estas investigaciones han sido estudios longitudinales enfocados al análisis de la progresión de minorías en la educación secundaria y superior, principalmente en Estados Unidos. En un número importante de investigaciones, los términos "retención" y "permanencia" se utilizan como dos caras de un mismo fenómeno, pareciendo no existir un claro consenso acerca de la definición y diferenciación de estos conceptos, como tampoco de la forma de medirlos. Mientras algunos autores definen la permanencia como la habilidad de los estudiantes para graduarse de un programa, otros lo hacen en términos de continuación u obtención de buenos resultados educativos (Tinto, 1993). ${ }^{4}$

Gran parte de la literatura plantea una amplia gama de factores explicativos asociados con la retención. Entre éstos se mencionan atributos personales de los alumnos, factores sociales y prácticas institucionales. Un foco tradicional de interés dentro de los estudios sobre permanencia educativa tiene relación con las características individuales o de personalidad de los estudiantes. Dentro de estas

4 Para efectos de esta investigación, utilizaremos los conceptos de permanencia, y/o retención como sinónimos. La razón de esta decisión es que, a la fecha, existe escaso consenso respecto de la diferencia de ambos conceptos y, por tanto, son tratados como fenómenos que refieren al mismo proceso. 
investigaciones (Goldfinch y Hughes, 2007; Catterall, 1998; Floyd, 1996; Mischel, Zeiss \& Zeiss, 1974) se destaca la importancia de la autoestima, el optimismo, autocontrol, confianza en las capacidades y perseverancia.

Adicionalmente se ha señalado que la confianza en la graduación -es decir, la convicción sobre la graduación- resulta ser un rasgo de estudiantes más exitosos. Los estudiantes vulnerables que permanecen son aquellos que confían en que van a lograr sus objetivos, más allá de las adversidades que encuentran en el camino (Baker $\&$ Siryk, 1984; Baker \& Schultz, 1992; Baker \& Siryk, 1989a). La literatura sugiere que estos estudiantes desarrollan conciencia de la adversidad, visualizando activamente las posibilidades de éxito.

De manera creciente, algunos estudios sobre permanencia han incorporado el concepto de "resiliencia" para entender la capacidad de permanencia de estudiantes vulnerables. Reed-Victor \& Stronge (2002) sostienen que la resiliencia de los jóvenes no significa que ellos no sean vulnerables o que estén lejos de resultar afectados por las adversidades que enfrentan, sino más bien que logran desarrollar y usar los recursos disponibles para sobreponerse a tales circunstancias.

En otras palabras, estudiantes que desarrollan conductas resilientes logran permanecer de manera exitosa en el sistema universitario, porque cuentan con una visión proactiva y consciente de las barreras y oportunidades que dicho sistema les ofrece (Masten, 1994). Si bien la utilización de la perspectiva de resiliencia es nueva en los estudios de educación superior (Warren, 2005; Henderson, 2003; Reese, 2000; Windfield, 1996; Miller, 1995; Connell et al., 1994), ha ido ganando creciente reconocimiento como contexto para examinar por qué sólo algunos estudiantes permanecen, mientras que otros con características sociales y económicas similares no lo hacen (Walker, 2006; O'Connor, 2001, 2002).

Algunas investigaciones han destacado la existencia de recursos que aminoran el impacto de los riesgos de deserción que enfrentan los estudiantes vulnerables, identificándolos como "factores protectores" 
56 RETENCIÓN DE ESTUDIANTES UNIVERSITARIOS VULNERABLES - Andrea Canales, Danae de los Ríos

(Fullana, 1998). La consideración de estos factores es especialmente importante, ya que desplaza el foco de atención desde el estudiante (es decir, su capacidad personal y perseverancia) hacia el contexto social en el que se encuentra inserto.

Centrar la atención en el contexto social e institucional resulta fundamental para comprender la retención, ya que permite comprender dicho fenómeno como el resultado de la interacción entre un estudiante y el medio en el cual se desenvuelve (Thomas, 2002, Tierney, 2001, 1999). Es decir, la permanencia de los estudiantes no puede ser entendida como una capacidad individual de determinados estudiantes, sino como el resultado de la interacción entre ellos y su contexto.

El papel que las instituciones de educación superior juegan en la progresión de estudiantes vulnerables ha sido una de las temáticas más tratadas en la investigación educacional y sociológica. Una de las formas más tradicionales para promover la permanencia de alumnos vulnerables ha sido el uso de apoyos financieros o becas. Sin embargo, como plantean Gladieux y Swails (1998), estas ayudas no son suficientes para asegurar su progresión.

En sociología, existe una larga tradición teórica y empírica que ha explorado aspectos organizacionales que impactan la retención estudiantil (Tinto 1975, 1993; Bean 1982; Astin, 1984; Pascarella y Terenzini 1991). Tinto es uno de los autores más reconocidos en este ámbito sugiriendo, entre otros, los efectos positivos de la interacción entre profesores y alumnos. Tinto (1987) plantea que mientras mayor interacción entre alumnos y profesores exista, mayores probabilidades de que los alumnos finalicen sus estudios.

Pascarella y Chapman (1983), por su parte, han señalado que el compromiso de los alumnos con sus instituciones tiene un efecto significativo en la permanencia de los estudiantes. Barr y Rasor (1999), por otra parte, sugieren que aquellos estudiantes que utilizan los servicios estudiantiles y se afilian a grupos o asociaciones permanecen más que aquellos que no hacen uso de estos servicios o conexiones. La permanencia de estudiantes en las instituciones está 
fuertemente determinada por las redes y relaciones sociales de los alumnos y, por ello, Hays y Oxley (1986) plantean que dichas redes son fundamentales en la adaptación e integración estudiantil.

La familia ha sido objeto de especial atención en los estudios sobre permanencia y retención. Jarret (1997) ha sugerido que los padres pueden actuar como apoyo, disminuyendo el riesgo de abandono. Por ejemplo, pueden ayudar a los jóvenes a desarrollar una alta autoestima y un sentido de eficacia educativa, a pesar de los obstáculos a los que se ven enfrentados. Dentro del papel jugado por las familias las expectativas son un factor relevante, pues los padres resultan ser una fuerza motivadora para ingresar a la universidad.

También ha sido descrita la función que desempeñan las familias en el desarrollo de "hábitos de estudio", ayudando a los estudiantes a desarrollar usos y prácticas educativas que facilitan el aprendizaje. Son importantes en la "definición de prioridades"; por ejemplo, las familias pueden actuar orientando a los estudiantes en las elecciones de asignaturas y programas educativos. Finalmente, pueden contribuir al desarrollo de valores relevantes como la puntualidad y la responsabilidad (Barnett, 2004), facilitando con ello la progresión de sus hijos en el sistema educativo.

En síntesis, la permanencia de estudiantes vulnerables no puede ser entendida únicamente como un fenómeno asociado a capacidades o atributos individuales, sino también como un proceso influenciado por el contexto social en el que estos estudiantes se desenvuelven. Familia, pares e instituciones educativas juegan un papel central en la progresión de los alumnos vulnerables y, por ello, es necesario incorporarlos al momento de diseñar iniciativas orientadas a mejorar las condiciones para la progresión exitosa de éstos.

\section{Marco metodológico}

Tomando en consideración los antecedentes expuestos en la sección precedente, los investigadores decidieron explorar el tema de la retención y permanencia de estudiantes vulnerables desde una perspectiva cualitativa. Para efectos de esta investigación, se seleccionó 
58 RETENCIÓN DE ESTUDIANTES UNIVERSITARIOS VULNERABLES - Andrea Canales, Danae de los Ríos

una muestra de estudiantes universitarios que tuviera al menos alguna de las siguientes características de vulnerabilidad:

i) pertenecer a los dos primeros quintiles de ingreso;

ii) pertenecer a grupos étnicos minoritarios;

iii) tener responsabilidades familiares (ser jefe de hogar o tener hijos), y/o

iv) trabajar más de 20 horas semanales.

En total, se realizaron 35 entrevistas a alumnos vulnerables de distintas universidades localizadas en cinco regiones del país (V, Metropolitana, VIII, IX y XI). Se entrevistó a 20 hombres y 15 mujeres de ocho universidades. Ocho de los entrevistados tenían hijos y 10 eran descendientes de algún grupo o minoría étnica. De los 35, 17 desarrollaban actividades laborales importantes (20 o más horas de trabajo). Con base en el autorreporte, todos los estudiantes calificaban como estudiantes vulnerables, es decir, pertenecían a familias pertenecientes a los dos primeros quintiles de ingreso.

Adicionalmente, se realizaron siete entrevistas individuales a encargados académicos o directores de asuntos estudiantiles, con el fin de conocer la mirada institucional respecto del problema de estudio. Específicamente, mediante estas entrevistas se buscó indagar sobre las visiones y estrategias institucionales que se ofrecen para dar soporte a los estudiantes vulnerables en el sistema educativo.

Dada la naturaleza exploratoria del estudio, la selección de casos privilegió la representación de distintas áreas de conocimiento e instituciones educativas tradicionales a través del territorio, buscando maximizar la heterogeneidad de casos. El uso de casos variados, es decir, provenientes de diferentes instituciones, localidades y áreas de conocimiento, proporcionó antecedentes de gran riqueza que permitieron comprender el contexto y las explicaciones que los estudiantes y sus instituciones dan a las experiencias de permanencia en el sistema de educación superior. Adicionalmente, se realizaron dos entrevistas grupales para ampliar la validez de los principales hallazgos. En ellas se convocó a estudiantes de universidades y carreras similares para facilitar el diálogo entre participantes. 
Tanto las entrevistas individuales como las grupales se realizaron siguiendo una pauta semiestructurada previamente testeada. Todas las entrevistas fueron grabadas y transcritas con el fin de facilitar el análisis de la información, realizado con un sistema de codificación abierta, buscando patrones temáticos relevantes de las entrevistas transcritas.

\section{Resultados}

La evidencia sugiere que los riesgos que enfrentan estudiantes vulnerables son variados, destacándose los que tienen relación con dificultades académicas, emocionales/afectivas, sociales y económicas. Estos problemas se combinan en distintos momentos de la vida universitaria de los alumnos, afectando su desempeño y vida académica al interior de las instituciones que los albergan.

Con relación a las dificultades académicas, los entrevistados señalan que la falta de hábitos de estudio y las deficiencias en el manejo de contenidos son problemas importantes en los primeros años de universidad. Manifiestan que éstos son un hándicap con el que deben vivir durante los primeros semestres de sus carreras y que, en determinadas situaciones, se arrastran hasta etapas avanzadas del programa de estudios.

"(Al principio) fue horrible, porque todos decían que era fácil y se enojaban conmigo porque yo no entendía y pensé en un momento que esto no era lo mío. Pero como me iba bien en otros ramos que me gustaban, entonces mis amigos me decían: 'cálmate si el ramo no va a durar para siempre'. Entonces mis deficiencias del colegio, que para algunos son nimiedades, yo aún las tengo, básicamente en la parte científica. Y siempre me pasa que voy más abajo que el resto en cuarto año de la carrera...".

La transición/adaptación a la universidad es un proceso estresante, pues los estudiantes se ven enfrentados a una complejidad cognitiva a la cual no han estado expuestos durante la etapa escolar. Las dificultades iniciales son percibidas como una situación 
60 RETENCIÓN DE ESTUDIANTES UNIVERSITARIOS VULNERABLES - Andrea Canales, Danae de los Ríos

altamente desafiante, al punto de hacerlos cuestionar sus habilidades y competencias académicas. Esto constituye para ellos un factor de riesgo, porque observan una gran distancia entre las estrategias de aprendizaje desarrolladas durante la etapa escolar y las requeridas en el mundo universitario. Tal como lo indica el siguiente entrevistado:

"No me costaba el colegio, y eso me ha jugado un poco en contra en la universidad, porque como en el colegio nunca me costó nada, no tenía hábitos de estudio. Acá tuve que comenzar a estudiar. Por ejemplo, en la primera prueba que tuve me fue súper mal porque me saqué un 3,0, y una niña me dijo: 'A mí también me fue mal, me saqué un 2,0. ¿Te leíste tal cosa? No, ¿y qué te estudiaste? El cuaderno'. Y ahí me di cuenta que no estaba estudiando bien y que tenía que cambiar los hábitos de estudio".

Los estudiantes perciben que las formas de aprendizaje adquiridas en la secundaria no se ajustan a las necesidades del sistema universitario, que demanda un trabajo sistemático. Más aún, la dificultad para desarrollar una capacidad esta clase de trabajo constituye un debilidad que algunos no logran superar durante la enseñanza superior.

“...Yo tengo muy pocos hábitos de estudio, he cultivado algunos pero casi nada, porque en el colegio se estudiaba la nada misma, ni en la enseñanza media, sólo usaba los cuadernos. Pero cuando llegué aquí me vi obligada a sentarme a estudiar, esa es mi pelea constante, si estudiar o ver televisión, tengo la motivación pero me cuesta...".

"Al final, cuando uno viene de liceos de verdad malos, en realidad no estudias, porque no tienes necesidad de hacerlo. Te dicen: 'vamos a tener prueba de biología' y lees el texto o la materia, lo poco que anotas y sabes lo que te van a preguntar por lo que no tienes necesidad de estudiar. En cambio (...) uno se da cuenta de que tiene compañeros que vienen de colegios particulares, a los que les enseñaron métodos de estudio, y yo creo que en eso radica la diferencia: ellos venían con técnicas de 
estudio, por ejemplo sabían destacar. De eso me daba cuenta yo, ellos sabían destacar, usaban colores, yo no sabía, porque nunca había estudiado; no había tenido la necesidad de estudiar. Uno tiene que darse cuenta y pedir ayuda. Conciliar tiempos también es complicado, pero hay que hacerlo".

Para los alumnos entrevistados, las dificultades económicas persisten a lo largo del proceso formativo. La situación laboral de sus familias tiende a ser frágil, existiendo dificultades permanentes asociadas con los bajos salarios o con la inestabilidad laboral de los adultos con los que conviven al interior de sus familias.

"...en mi casa el dinero únicamente es para comer y para pagar cuentas (...) es como un círculo vicioso porque pedimos préstamos y al mes siguiente tenemos que pedir otro para pagar el anterior y nunca sales (...) el tema económico sí ha sido un problema para mí, más allá de las becas que tenga".

"(Mi mayor problema en la universidad) han sido las apreturas económicas. Eso de repente de atrasarse en las cuotas, de que te estén molestando que estás atrasado, mandándote mails 'está atrasado'. Pero nunca he caído en un asunto de cobranza, ni mi familia. Pero yo creo que el asunto económico, eso de no disponer es una preocupación. Al principio sí influyó en mis estudios, era una preocupación constante. Pero después no, porque la mentalidad ha sido sacar adelante como fuera el asunto (refiriéndose a los estudios)".

Para aminorar las dificultades económicas y a medida que avanzan en sus respectivos programas, muchos desarrollan alguna actividad laboral a tiempo parcial en forma más sistemática. Los ingresos obtenidos les permiten pagar gastos asociados a sus estudios, como acceder a internet, comprar un computador, sacar fotocopias, movilización, entre otros.

"Desde finales del 2005 que estoy haciendo clases particulares. O lo que salga. La hora sale más o menos 8 mil pesos, en esos tiempos, 7 mil pesos. Y uno pilla 
62 RETENCIÓN DE ESTUDIANTES UNIVERSITARIOS VULNERABLES - Andrea Canales, Danae de los Ríos

unas 3 clases por mes. 21 o 24 mil pesos en este caso. Pero tú tienes que pagarle el $10 \%$ a la universidad. O sea, al final, yo saco mensualmente más o menos \$25.000, porque puedes tener a veces una clase más, una clase menos. \$ 25.000 mensuales. Que para mí es harto. Por eso cuando me llama mi mamá y me dice, 'no tengo para ponerte en el banco', yo le digo no, no te preocupes, yo tengo ahora"

Las dificultades de estos estudiantes no se limitan a la falta de recursos monetarios. En algunos casos, se ven enfrentados a experiencias familiares o sociales límite que afectan negativamente su progresión educativa, tales como situaciones de hacinamiento en sus hogares, delincuencia en sus barrios y otros problemas que hacen extremadamente dificultosa su progresión educativa.

"Mi hermana se casó y mi cuñado ha estado tres veces preso por tráfico. Mi otra (hermana) se metió con un drogadicto y pasa puro sufriendo y tiene puros dramas. (...) Hasta ahora sigo preocupado, porque ella está saliendo de una audiencia en (Salamanca) donde está viviendo. Mi hermana se viene a Chile, se va a divorciar y siempre son puros problemas. Imagínate estar (durmiendo) con mi hermana, mi cuñado, más la guagua. (Por eso) no me iba bien porque dormía mal y en las mañanas despertaba mal y me levantaba mal y pegaba un portazo y me iba. De hecho tuve un colapso nervioso después que entre a la universidad (...) Me vino como un bajón, cuando desperté en la mañana eran vueltas y vueltas, no me podía parar, me vino un colapso en el oído medio igual al que le vienen a las mujeres pero era por estrés y llegué a llorar de desesperación porque no oía nada y de repente se me pasó (...) fue un asunto completamente nervioso, colapso nervioso. Pero eso ya pasó y mis hermanos se fueron de la casa".

Los problemas de integración educativa son otro escollo que los estudiantes vulnerables deben enfrentar al momento de ingresar a las instituciones de educación superior. Para muchos la universidad constituye un espacio social muy distinto al de la educación secundaria. En particular en instituciones de elite, los 
alumnos vulnerables se sienten especialmente distintos al resto de sus compañeros. El proceso de integración resulta arduo porque los estudiantes desconocen la vida universitaria y sus requerimientos. La universidad supone un proceso de adquisición y adaptación a nuevos códigos culturales y simbólicos (Canales y De los Ríos, 2007).

"Son mundos súper distintos. Tú entras a la universidad y son todos súper cuicos, y eso es súper estresante. Por ejemplo, cuando vas a hablar en clases, o al principio cuando ibas a hacer alguna referencia preferías no hacerla por miedo, porque era súper violento escuchar hablar al resto en su entonación de clase, mientras que uno todavía tiene una entonación más 'flaite"'.

El acceso a la universidad es para muchos de estos estudiantes un primer encuentro con el sistema de educación superior, ya que gran parte de ellos son primera generación de sus familias en acceder al sistema universitario. Se produce, por tanto, un desajuste de expectativas derivado del desconocimiento de la vida universitaria y sus implicancias.

Factores como el alejamiento del hogar, el desconocimiento de la ciudad o zona en donde estudian, los largos trayectos de traslado, la pérdida de grupos de referencia y la necesidad de generar nuevas amistades y grupos de trabajo académico son un desafío para ellos. Pueden llegar a experimentar soledad y angustia durante este periodo, sin que necesariamente existan los soportes institucionales para enfrentarlas.

"Me afectó el no tener una residencia en Santiago. De repente (no) poder decirle a mis amigos: 'sabes que no tengo dónde quedarme a estudiar'. O sea, si alguien me dice, quedémonos a estudiar, yo me tendría que quedar en tu casa. Es decir, yo no puedo venir porque no tengo donde quedarme".

"Uno igual esperaba encontrarse con gente similar, pero uno se encuentra con gente muy poco tolerante. Me ha sido muy difícil mantenerme en la carrera, sobre todo por la soledad, echar de menos a mi mamá. Me costó 
64 RETENCIÓN DE ESTUDIANTES UNIVERSITARIOS VULNERABLES - Andrea Canales, Danae de los Ríos

mucho vivir sin ella, en ese sentido dependo mucho de ella. Lo otro son las distancias de la ciudad y el tiempo que te toma la universidad, que es muy frustrante y yo creo que hay muchos periodos de depresión que uno los supera por el solo hecho de pensar que tienes que terminar la carrera, pues de lo contrario todo el esfuerzo realizado ha sido en vano...".

A pesar de las dificultades de adaptación, los estudiantes enfrentan los problemas de integración con un gran sentido de orientación al logro. La meta de terminar un programa de estudios y obtener un título profesional es un aliciente fundamental para permanecer en la universidad. Los alumnos vulnerables que permanecen en el sistema también presentan características destacadas de perseverancia, esfuerzo y seguridad.

Si bien muchos reconocieron incertidumbres iniciales respecto de sus capacidades -especialmente cuando obtuvieron bajas calificaciones o reprobaron algunos cursos en los primeros años- éstas no llegaron a traducirse en una clara intención de abandonar los estudios. Treinta y tres casos del total de entrevistados, han desarrollado sus programas académicos sin quiebres o interrupciones.

"El primer año reprobé cuatro ramos, porque el cambio fue muy fuerte. En el colegio no estudiaba, con lo que aprendía en clases me bastaba para que me fuera bien y luego llegué acá donde todos estaban dispuestos a estudiar, a sacarse la mugre (...) Nunca sentí que esto no era lo mío, me sentía bastante agradado acá (en la universidad), lo pasaba bien y uno de los principales motivadores es cómo tú te sientes y yo me sentía bien haciendo lo que pensaba llegar a ser, entonces no me hacía problemas. No pensaba que no sirvo, porque un montón de gente puede pensar así o la gente más insegura podría pensar que no sirve si reprueba (...) Yo en cambio pienso que hay algo que no sé y como no lo sé tengo que aprenderlo (...) Para mí (reprobar) era simplemente un proceso de entrenamiento, yo acá a los chicos en la escuela les digo que les falta entrenamiento, ustedes no se pueden echar a morir porque no saben, $\mathrm{o}$ 
sea, es tonto (...) no puedes decir no sé y me voy (...) tú no tienes porque saber (...) tu vienes acá a estudiar a aprender".

La confianza en sus capacidades y la perseverancia parecen estar fuertemente relacionadas con claras metas y propósitos que los motivan a permanecer en la universidad. La gran mayoría manifestó que, a pesar de las dificultades, siempre han tenido claras sus metas y las motivaciones por las que continúan estudiando.

"Me superé reprobando ramos, pasando sustos, sacrificándome. Por ejemplo, cuando trabajaba y tenía que llegar a estudiar a las nueve o diez, entonces tenía que ser resistente nomás".

Otro rasgo que caracteriza a estos estudiantes es el conocimiento de sus límites y posibilidades (autoconocimiento), así como una clara racionalidad en la toma de decisiones, considerando sus habilidades y el contexto en el que se desenvuelven. Anticipan los beneficios asociados a la obtención de un título profesional y por ello tienen la disposición a enfrentar las dificultades asociadas al proceso formativo.

"Yo tenía claro que tenía que estudiar. Yo soy súper malo para hacer trabajos físicos, soy súper torpe. En cambio mi hermano no, el anda metido con fierros, mecánicos, es creativo, inventa cosas. Yo no, prefiero mil veces estar leyendo un libro que andar metido soldando, aunque igual me gustaría hacerlo porque lo encuentro súper práctico, pero no me resulta fácil, no lo puedo hacer. Además, sabía que si no estudiaba iba a tener que estar barriendo o haciendo trabajos no calificados y ganando súper poca plata. Más que nada por miedo al hambre me metí a estudiar a la universidad".

Como se mencionó, los estudiantes vulnerables tienen claras aspiraciones vinculadas al mejoramiento de sus condiciones de bienestar. La obtención de un título profesional implica mejorar sustantivamente su calidad de vida, incorporando mayor seguridad y estabilidad económica. Es posible afirmar que asocian la obtención 
66 RETENCIÓN DE ESTUDIANTES UNIVERSITARIOS VULNERABLES - Andrea Canales, Danae de los Ríos

de un título profesional con movilidad social ascendente o, en otras palabras, con el mejoramiento de sus condiciones de vida respecto de la de sus padres. Para algunos de los entrevistados, la obtención de un título profesional está asociada incluso a la superación de la pobreza en la cual se encuentran insertas sus familias.

“.. Yo pienso (la educación) por el lado de la estabilidad, de poder tener un trabajo más estable y digno. Por ahí es donde lo veo (...) y a veces, cuando uno se va en un análisis más profundo, me pregunto cómo puedo contribuir mejor a la sociedad, porque si quieres ser un aporte debes saber y tener ciertos objetivos claros, y que a veces no son todos realizables, por el sistema en que nos encontramos hoy en día...".

En un segundo nivel existe una motivación por realizar una contribución a la sociedad o comunidad en la cual están insertos. El interés de la mayoría de los entrevistados es "devolver" o "llenar de orgullo" a sus familias y comunidades (en los casos de alumnos indígenas). La educación en ese horizonte es percibida como un reconocimiento a la familia, que se visualiza como un recurso primordial en el proceso formativo.

"...cuando termine mi carrera quiero ver sus caras de felicidad, me gusta lo que hago, pero el incentivo primordial es ver a mi mamá, mi vieja que no sabe nada lo que me pasa en la universidad....".

Una de las características de personalidad con que cuentan los alumnos vulnerables que logran progresar en el sistema universitario, y que resultó ser uno de los hallazgos más interesantes de esta investigación, es la clara conciencia de su vulnerabilidad y de los riesgos a los que están expuestos, dadas sus características socioeconómicas. Los entrevistados manifestaron que la posibilidad de estudiar en la universidad es única en sus vidas y que no debe ser desaprovechada.

“...Yo tomé la decisión de quedarme en primero (...) y hay que echarle para delante pues sabes todo lo que implica retirarse. Aquí te das cuenta que los que tienen 
esta oportunidad no pueden darse el lujo de retirarse, no nosotros...".

"Esta es la oportunidad, porque si yo no estudio, tendría solamente que trabajar...".

La conciencia de vulnerabilidad también se expresa en el reconocimiento y acercamiento a otros alumnos que comparten características "sociales" similares en las instituciones educativas. Los entrevistados manifestaron haber desarrollado estrategias con estos pares para enfrentar sus dificultades académicas y de integración social en las instituciones educativas.

"Yo me hice muy amigo de un estudiante de astronomía que venía de Arica y era poseedor de todas las posibles becas de este país, hasta una beca Coca Cola tenía. Él tenía una teoría, que decía que en la universidad los polos 'flaites' se atraían. Y justo cuando yo me estaba cambiando de carrera él también estaba en eso, y hasta el día de hoy somos súper amigos. A los dos nos ofrecieron la ayudantía de investigación, nos fuimos potenciando mutuamente. Llegábamos a las 8 de la mañana y nos íbamos a las 21:00 hrs. de la universidad (...) Y en todos los ratos intermedios que podíamos estudiábamos. Y ese factor fue muy importante para mí”.

Podríamos señalar que los alumnos vulnerables tienen conciencia de constituir un grupo social distinto al interior de las instituciones educativas. Tanto los de nivel socioeconómico bajo como aquellos que proceden de comunidades étnicas señalan sentirse muy distintos respecto de sus pares, reconociéndose como minorías al interior de las instituciones educativas.

"Llegué y la primera clase preguntaron cuántos de los que estábamos ahí tenían papás ingenieros y más de la mitad levantaron la mano. Yo pensaba que la universidad era distinta, más diversa, pero igual tiene una brecha socioeconómica alta. Entonces me costó tolerar el comportamiento un tanto infantil de mis compañeros en primer año. Muchos seguían como en el colegio pagado. Yo estaba acostumbrada a tener que portarme bien, casi 
68 RETENCIÓN DE ESTUDIANTES UNIVERSITARIOS VULNERABLES - Andrea Canales, Danae de los Ríos

sin toser, y aquí conversaban. Yo estaba acostumbrada a cosas más rigurosas (...) Los temas de verdad eran muy diferentes. Ellos contaban cuando fueron a Disneylandia y yo cuando fui a Fantasilandia. Entonces los temas domésticos que uno conversa y se ríe son distintos (...) No sé, por ejemplo, ellos nunca han ido a comprar el pan a un almacén. Va la nana a comprarles al súper...”.

La mayoría de los entrevistados señaló que el apoyo de sus pares resultó crucial principalmente durante sus primeros años de estudios universitarios. Con ellos desarrollaron relaciones sociales basadas principalmente en la solidaridad, apoyo y confianza mutua, orientadas a enfrentar sus problemas académicos y sociales. Específicamente, frente a las dificultades académicas, los estudiantes desarrollaron estrategias de estudio colectivo, discusión, lectura y síntesis. Asimismo, sus pares resultaron ser un soporte "social" significativo, es decir, un referente que les ha permitido sentirse más partícipes e integrados a sus instituciones.

"Construíamos metodologías de estudio en conjunto. Un día nos compramos una grabadora y uno de los dos transcribía las clases, y lo mismo con las ayudantías, porque tenían la estructura de la pauta de respuesta de la pregunta. Nos intercambiábamos las cosas, hacíamos resúmenes, nos dimos cuenta que nos rendía más estudiar en las mañanas que en la noche. Nos íbamos viendo los puntos ciegos y nos dábamos feedback. Entonces, no fue casualidad que a nosotros dos como equipo nos dieran esta ayudantía, y después como equipo también nos suben a un proyecto Fondecyt (...) Fue una relación que dio muchos frutos y que hasta el día de hoy los da. Tenemos una dinámica de lectura en que nos juntamos todos los viernes a leer".

Se desprende de las entrevistas que los alumnos vulnerables se sienten comprometidos con sus programas de estudios e instituciones. Estos resultados son coincidentes con otras investigaciones que muestran que el sentido de pertenencia, compromiso e integración son factores fundamentales para explicar su permanencia y retención en el sistema educativo. 
Las características de personalidad, sin embargo, no son los únicos factores que explican la permanencia de estudiantes vulnerables en la educación superior de tipo universitaria. Es el resultado de la combinación de capacidades personales, presencia de redes sociales positivas, soportes familiares, así como también soportes ofrecidos por las instituciones.

De manera concordante con lo señalado por la literatura, los hallazgos sugieren que existe una relación directa entre la permanencia de los estudiantes vulnerables y el apoyo que les brindan sus familias: entregan soporte afectivo y de contención en momentos difíciles de sus vidas universitarias.

Un número mayoritario de entrevistados sugiere que el apoyo de ellas resultó fundamental, ya que actuaron disminuyendo el estrés asociado a lidiar con los "fracasos" de las primeras calificaciones y reprobaciones. Tal como lo expresa el siguiente entrevistado, actúan como ancla o agentes moderadores de la conducta, evitando visiones extremas respecto de éxitos y fracasos.

“...Mis papás siempre han sido un apoyo súper importante, mi hermano también. Yo creo que mi familia me ha salvado. Es súper importante tener una familia como pilar, que esté atrás que te diga que no te hagas problemas, que te dé ánimo, porque te ayuda a sobrellevar de mejor manera los fracasos y a mantener los pies sobre la tierra cuando tienes éxitos. No sacas nada con puros elogios, te pueden decir 'OK, te ha ido bien, pero aterriza', y lo mismo cuando andas bajoneado, 'oye, pero tú no estás tan mal, piensa que hay gente que está mucho peor'...”.

Los resultados sugieren que la familia -especialmente los padres- tienen un rol activo en la socialización inicial de sus hijos, promoviendo valores y actitudes como el esfuerzo, la perseverancia y el logro de metas de largo plazo. Los estudiantes valoran positivamente dicha socialización y reconocen la importancia de estos valores en su permanencia en la educación superior. 
70 RETENCIÓN DE ESTUDIANTES UNIVERSITARIOS VULNERABLES - Andrea Canales, Danae de los Ríos

"Yo siempre fui perseverante y paciente, y no pienso que debo retirarme cuando hay soluciones. Tengo compañeros que tienen millones de deudas y saben que después van a poder hacerse cargo de eso. Yo creo que (la universidad) es perseverar y paciencia, e idealmente tener el apoyo moral de los padres porque si no se tiene un apoyo anímico importante es súper difícil. De repente a uno le puede ir mal en un control y estar achacado, pero el apoyo y el amor de la mamá siempre están. Mis padres siempre me han dicho que si ellos no están o mis hermanos no están, yo debo terminar la universidad. Tendría que pasar algo muy terrible para que yo abandone la universidad".

Por otra parte, aquellos alumnos que contaban con familiares (hermanos, primos, tíos) con algún tipo de experiencia en la educación superior señalaron que su apoyo resultó especialmente orientador y útil en su ingreso a la universidad. El conocimiento de experiencias directas, a través de hermanos, primos u otros, les permitió sentirse mejor preparados y familiarizados con los desafíos académicos y extra-académicos que la vida universitaria supone.

"Mi papá pasó por un periodo de universidad y me habló de problemas en la universidad. A mí me ha tocado contarle los míos y quizá no los hubiera superado si no me hubiera aconsejado. En el caso de las becas, por ejemplo, yo no me hubiera enterado de las becas si él no me las hubiera comentado, o también cuando me sugería que no me llevara más de tres libros para la casa porque no me los iba a estudiar. Por tanto, sí me ha ayudado mucho...".

Tan relevantes como los padres y grupos de referencia resultan los soportes proporcionados por el sistema de educación superior y por las instituciones educativas. Las ayudas financieras (créditos y becas) ocupan un lugar central dentro de estos soportes. Todos los entrevistados que participaron en este estudio manifestaron contar con diversas ayudas estudiantiles entregadas por el Estado y las propias instituciones educativas. 
"Yo tengo la beca Presidente de la República que es de como $\$ 42.000$ pesos. Con esa plata yo me he podido comprar ropa, esa plata me ha servido mucho para sacar fotocopias, para movilizarme. Incluso un tiempo me sirvió para pagarme un medicamento muy caro y también para ayudar en mi casa cuando no hay plata para pan".

"Ellos te dan la plata, tú ves en qué la ocupas. También hay becas de alimentación, tú decides cuál te conviene más. El alumno de pregrado gasta mucha plata en fotocopias, más que en almorzar y se dieron cuenta de eso e hicieron una beca de fotocopias. Y lo bueno es que la escuela tiene hartos recursos dando vuelta y con ellos se trata de ayudar al alumno...".

Los recursos y modalidades de becas, no obstante, varían entre las instituciones universitarias. Algunas cuentan con una amplia diversidad, que cubren aranceles, movilización, alimentación, fotocopias y medicamentos; mientras otras sólo proporcionan becas de arancel y matrícula. Las ayudas estudiantiles son manejadas a través de oficinas centralizadas y, de forma complementaria, algunas facultades o departamentos cuentan con recursos especiales que complementan los beneficios centrales. Sin embargo, esta situación se limita a un número restringido de instituciones.

Junto con las ayudas estudiantiles provistas por el Estado y las propias instituciones, los estudiantes entrevistados destacaron que sus universidades también contaban con soportes institucionales, como servicios médicos, sicológicos y dentales. Se destaca la presencia de estos servicios, principalmente en instituciones universitarias tradicionales.

"Todos mis compañeros se están haciendo tratamiento dental, es súper bueno. Imagínese que un compañero se lo hizo afuera y le salió 300 mil y acá sale por 80 mil. Se habla con la asistente social y le prestan el dinero y le pregunta en cuántas cuotas lo quiere pagar. Van y pagan y se hacen el tratamiento completo y después pagan el 
72 RETENCIÓN DE ESTUDIANTES UNIVERSITARIOS VULNERABLES - Andrea Canales, Danae de los Ríos

préstamo, es buenísimo, hay ginecólogo para las mujeres, médicos generales, terapeuta, hay de todo. Eso me parece buenísimo, la mayoría de las cosas son gratis y si no te prestan la plata y te dan facilidades de pago".

Si bien las instituciones universitarias estudiadas concentran sus esfuerzos en proporcionar ayudas financieras y sociales a los alumnos, existe un ámbito de soporte, menos desarrollado y altamente demandado por los estudiantes, que se refiere a los recursos docentes y de infraestructura. Los jóvenes entrevistados reconocieron hacer uso intensivo de ellos, señalando que constituían un aspecto central para explicar su permanencia en el sistema universitario.

La mayoría de los entrevistados destacó que sus universidades les proveían de lugares de estudio, bibliotecas y recursos computacionales para desarrollar sus actividades académicas. Para la gran mayoría resulta fundamental hacer uso de estos servicios, ya que en sus hogares no contaban con las condiciones necesarias para realizar sus actividades académicas.

"No sé cómo será en otras facultades, pero aquí en ingeniería tienen súper buenas instalaciones. Los computadores son bacanes. En la casa igual tengo, pero como somos tantos siempre está colapsado. En cambio aquí te dan una cuota de papel y aparte tengo otra cuota de papel por el departamento...".

"Mi casa es de madera, al lado escuchan reggaetón desde las 21:00 hrs. hasta la 01:00 de la mañana, por lo tanto es imposible estudiar ahí...".

Una de las falencias detectadas en esta investigación se relaciona con la disponibilidad y los horarios de acceso a los recursos de aprendizaje. Los horarios en que están abiertas las bibliotecas y salas de computación no se adecuan necesariamente a los requerimientos académicos de los alumnos, especialmente en periodos de exámenes o al cierre de los trimestres o semestres.

Las universidades cuentan con sistemas de administración de recursos docentes que muestran poca flexibilidad frente a las 
demandas de uso de distintos grupos de estudiantes vulnerables. Por ejemplo, para los estudiantes que trabajan y tienen horarios más extendidos resulta especialmente problemático el acceso a estos recursos docentes.

"En mi facultad igual es problemático porque somos muchas personas, casi 200 por generación que quieren ocupar computadores que en definitiva se hacen poco. De repente quiero ver una nota y no se puede. Ahora tengo computador con internet y ya no tengo ese problema pero cuando entré y en clases te decían que teníamos que descargar las guías era terrible porque los computadores de la facultad eran muy lentos y toda la gente partía, pero lo que me chocaba era que te dijeran lo 'subimos a internet' pero yo me decía, 'no todos tenemos internet'. Y cuando tenemos que estudiar mucho no nos podemos quedar en nuestra biblioteca porque por horario nos echan...".

La evidencia de otros países sugiere que los apoyos institucionales deben desplegarse en distintos ámbitos de acción, dada la multidimensionalidad de desafíos que enfrentan estudiantes de mayor vulnerabilidad. En este contexto, resultan especialmente importantes las actividades de preparación y nivelación académica, tutorías o seguimiento a la progresión de los estudiantes.

Los resultados de nuestra investigación sugieren que los soportes académicos orientados a estos propósitos son escasos y poco sistemáticos. La evidencia muestra que estas iniciativas no son una práctica habitual en el contexto universitario estudiado. Cuando existen programas de apoyo, estos resultan ser iniciativas específicas -de pequeña escala- que no se insertan dentro de una política académica global institucional. Implícita o explícitamente, las instituciones universitarias operan con el supuesto de que los estudiantes podrán - dada su capacidad-compensar los vacíos formativos que traen de la educación secundaria.

El tratamiento de las dificultades académicas iniciales no parece entenderse como una labor de las universidades, sino más bien como un asunto de responsabilidad y esfuerzo individual. Bajo 
esta mirada, las instituciones educativas esperan que sean los propios estudiantes los que identifiquen sus debilidades académicas y la forma de superarlas. Esto, sin embargo, parece una exigencia desmedida para quienes están iniciando su proceso formativo.

Sólo en dos de las ocho instituciones estudiadas se pudo constatar la existencia de cursos de nivelación y preparación académica. Éstos fueron bastante valorados por los entrevistados, quienes destacaron que estas iniciativas les han permitido compensar los vacíos educativos arrastrados desde la secundaria, reduciéndose en parte las diferencias con aquellos que han recibido una educación secundaria de mejor calidad.

"Me sirvió hacer el curso de verano, no sólo por el hecho de adelantar un ramo, sino como para tomarle el peso a lo que te enseñaban, pues me di cuenta de todo lo que me faltaba, ahí conocí la diferencia abismante que podía tener con el resto. Entonces, con mucho esfuerzo, me fui poniendo al día...".

Un escaso número de instituciones universitarias ofrece tutorías académicas a sus alumnos. Cuando se desarrollan este tipo de estrategias, se observa un trabajo personal con los estudiantes -a través de estudiantes avanzados o profesores- que los orientan respecto de materias específicas (contenidos) o asuntos vocacionales. La mayoría de los estudiantes sugirió la importancia de contar con soportes en este ámbito, especialmente en lo que respecta al monitoreo de la progresión académica y orientación vocacional.

"Yo creo que más apoyo en cuanto a tutorías; preocuparse más de los alumnos que van mal, preocuparse de por qué un alumno tiene rojos siempre, o de por qué a veces no viene. Ojalá que tengan un asistente, como algunos lo tienen, que se preocupe de aquellos jóvenes que reprueban. A lo mejor se debe a problemas motivacionales y puede que necesiten ayuda en ese sentido, porque a veces, a mitad de carrera, uno se da cuenta de que no es lo de uno y o te va bien o te va mal...". 
La evidencia sugiere que los alumnos de mayor vulnerabilidad requieren soportes y monitoreo académico permanente, con el fin de reconocer sus necesidades e implementar estrategias que los ayuden a progresar en el sistema educativo.

\section{Visión institucional sobre la vulnerabilidad estudiantil}

Si bien los resultados de este estudio dan cuenta fundamentalmente de la visión de los propios estudiantes vulnerables respecto de sus experiencias en las instituciones educativas, queremos analizar brevemente la visión que las propias instituciones tienen sobre este problema.

La información recabada a partir de las entrevistas con personas que dirigen programas de soporte estudiantil no permitió dilucidar un discurso coherente en el ámbito institucional respecto al tratamiento de los distintos problemas que afectan a los alumnos vulnerables. Más allá de la entrega de beneficios concretos, no se observa una visión coherente orientada a la generación de oportunidades que permitan mejorar los niveles de permanencia de estudiantes que poseen una mayor vulnerabilidad.

Las entrevistas sugieren que la existencia de estas iniciativas no implica necesariamente una definición institucional sobre materias de equidad educativa. En otras palabras, la mayoría de ellas no se insertan claramente dentro de una estrategia global, política o compromiso institucional orientado a la búsqueda de progresiones educativas más equitativas entre estudiantes con distinto background social.

Se debe señalar que estas instituciones concentran gran parte de sus esfuerzos en entregar soportes económicos a los estudiantes vulnerables, no entendiéndose a cabalidad las otras necesidades de carácter académico, cultural y social que tienen estos estudiantes. Las estrategias implementadas privilegian la satisfacción de condiciones mínimas de operación estudiantil, sin avanzar hacia otros ámbitos relevantes de acción. Más claramente, es necesario definir estrategias de soporte más comprensivo para los estudiantes. 
76 RETENCIÓN DE ESTUDIANTES UNIVERSITARIOS VULNERABLES - Andrea Canales, Danae de los Ríos

Por otra parte, la evidencia sugiere que son limitadas las iniciativas tendientes a evaluar y perfeccionar los programas de soporte en operación, desconociéndose su impacto en la permanencia de los estudiantes de mayor vulnerabilidad. De las entrevistas analizadas se infiere que las iniciativas de evaluación a dichos programas resultan ser discretas, ya que en muchos casos son realizadas por los propios implementadores o no tienen continuidad en el tiempo.

\section{Conclusiones y recomendaciones}

Los resultados de esta investigación sugieren que la retención y permanencia de estudiantes vulnerables en la educación universitaria se explica por factores de carácter institucional, social, familiar y personal. Los alumnos que logran progresar en el sistema universitario hacen uso efectivo de la mayoría de estos soportes o recursos para hacer frente a las dificultades que enfrentan dentro del sistema educativo. La interacción entre capacidades personales, apoyos familiares, recursos sociales (redes) y soportes institucionales permite explicar su permanencia en la universidad.

Entre las capacidades personales que caracterizan a los estudiantes vulnerables que permanecen en el nivel universitario se cuentan factores como la perseverancia, esfuerzo, seguridad en sí mismos, definición de metas claras y propósitos asociados al proceso educativo. La decisión de estudiar adquiere en estos estudiantes un sentido racional, justificado en la búsqueda de seguridad y estabilidad económica.

Esta búsqueda resultó ser una fuerza motivadora para permanecer tras un título o grado académico. No se destaca en cambio una motivación esencial con las carreras escogidas, siendo percibidas como un medio para acceder a mayores niveles de bienestar. De forma concordante, todos los alumnos entrevistados manifestaron que estudiar una carrera en la universidad era una posibilidad única y que no podía ser desaprovechada.

Los estudiantes mostraron una clara conciencia de su vulnerabilidad y de los riesgos que han debido enfrentar para 
permanecer en el sistema universitario. Asimismo, tienen conciencia de constituir un grupo minoritario al interior de las instituciones educativas, principalmente en comparación con los alumnos provenientes de medios más acomodados (sectores medios, altos y de colegios particulares).

Manifestaron sentirse en desigualdad de condiciones respecto de sus pares en materias académicas, haciendo referencia al manejo de contenidos, hábitos de estudios, dominio de idiomas, redacción y cultura general. De forma similar, experimentaron, con mayor o menor intensidad, desigualdades en su capital social, vinculadas a la existencia de redes poco vinculadas al sistema universitario. También destacan una falta de recursos (computadores, internet, libros, vestimenta, entre otros) para desempeñarse adecuadamente dentro de sus instituciones y programas.

En relación con el papel jugado por la familia, los resultados de este estudio sugieren que ésta puede brindar un importante soporte afectivo y de contención emocional entre los estudiantes de mayor vulnerabilidad social. Esto es particularmente relevante durante los primeros años de estudio, pues durante este periodo los estudiantes entrevistados han enfrentado los momentos más difíciles de sus trayectorias académicas. El estudio también sugiere que la familia juega un papel fundamental en la socialización inicial (vinculado con la motivación por el aprendizaje y la superación) y en el cultivo de ciertos valores como el esfuerzo y la perseverancia.

Por otra parte, los hallazgos muestran que las instituciones educativas juegan un papel significativo en la permanencia de alumnos vulnerables, al proporcionar ayudas económicas relevantes a través de becas y créditos. Sin embargo, estos esfuerzos o soportes no se enmarcan dentro de una estrategia o política integral de bienestar estudiantil. Lamentablemente, las instituciones educativas analizadas tienen una visión parcial y frecuentemente limitada a las necesidades financieras de los estudiantes vulnerables, no reconociendo cierta heterogeneidad y diversidad. 
78 RETENCIÓN DE ESTUDIANTES UNIVERSITARIOS VULNERABLES - Andrea Canales, Danae de los Ríos

Las políticas institucionales para dar soporte amplio (más allá de lo estrictamente económico) son mínimas o inexistentes. Por ejemplo, la evidencia demostró que la gestión de los recursos docentes y de infraestructura no satisface adecuadamente las necesidades o requerimientos particulares de este segmento de estudiantes. En todos los casos estudiados, estos servicios se administraban para atender a un estudiante genérico, sin conocer las demandas particulares de diversos segmentos estudiantiles.

Se observa una falta de evaluación de las políticas académicas e institucionales, desde la perspectiva de los estudiantes de mayor vulnerabilidad. ¿Cuál es la política institucional hacia estudiantes de mayor vulnerabilidad? ¿Qué necesita un estudiante vulnerable para progresar exitosamente dentro de una universidad? Cada institución podría hacer estas preguntas al momento de evaluar sus servicios y recursos académicos e institucionales, clarificando con ello su compromiso con la equidad educativa.

Resultó más frecuente la aproximación de carácter asistencial hacia los estudiantes de mayor vulnerabilidad, sin buscar la forma de poner los recursos académicos e institucionales existentes al servicio de estudiantes cada vez más heterogéneos. En ese contexto, resulta esencial desarrollar una gestión integral de los soportes institucionales disponibles con el fin de promover la progresión de todos los estudiantes, reconociendo una multiplicidad de necesidades y formas de satisfacerlas.

Una mirada de mayor equidad educativa debiera incorporar fórmulas compensatorias más novedosas que permitan hacer frente en forma efectiva a los desafíos de la formación inicial de los estudiantes de mayor vulnerabilidad, así como también proporcionar soportes que faciliten su egreso y titulación. Sería en extremo perjudicial que estos estudiantes, que logran acceder a la educación superior, deban enfrentar grandes obstáculos en su trayectoria académica, sin que las instituciones y el sistema educativo que los acoge se hagan cargo de algunos de dichos obstáculos o barreras. 
La definición de estrategias y políticas de retención de estudiantes de mayor vulnerabilidad en el plano institucional debiera considerar un seguimiento sistemático de éstos, monitoreando de cerca sus necesidades académicas y extraacadémicas. Hasta ahora, se observa un conocimiento limitado de ellos, pues las universidades recopilan antecedentes sociodemográficos básicos, desconociéndose en la práctica sus problemas y necesidades.

De forma concordante con lo planteado en otros países, las instituciones debieran avanzar en forma más decidida en la implementación de sistemas de apoyo académico, como tutorías, nivelación de estudios y evaluaciones periódicas por parte de los propios alumnos y profesores, en materias vinculadas a sus necesidades económicas, afectivas y de integración social. En la medida en que las universidades desarrollen capacidad para monitorear las necesidades de sus estudiantes, podrán dar una mejor respuesta a sus necesidades educativas y, por tanto, se promoverán formas más profundas de equidad educacional.

\section{Referencias bibliográficas}

Astin, A. W. (1984) Student involvement: A developmental theory for higher education. Journal of College Student Personnel, 25, 297-808.

Baker, R. W. \& Siryk, B. (1984) Measuring adjustment to college. Journal of Counseling Psychology, 31, 179-189.

Baker, R. W. \& Siryk, B. (1989) Student Adaptation to College Questionnaire (SACQ). Los Angeles, CA: Western Psychological Services.

Baker, R. W. \& Schultz, K. L. (1992) Experiential counterparts of test-indicated disillusionment during freshman adjustment to college. NACADA Journal, 12, 13-22.

Barnett, Marina (2004) A Qualitative Analysis of Family Support and Interaction among Black College Students at an Ivy League University. The Journal of Negro Education, 73(1), pp. 53-68.

Barr, J. \& Rasor, R. (1999) Freshmen Persistence As Measured by Reaching Academic Achievement Benchmarks. Presentation at the Annual Conference of the Research and Planning Group for California Community Colleges. Lake Arrowhead, CA. 
80 RETENCIÓN DE ESTUDIANTES UNIVERSITARIOS VULNERABLES - Andrea Canales, Danae de los Ríos

Bean, J. (1982) Student attrition, intentions and confidence: Interaction effects in a path model. Research in Higher Education, 14, pp. 425-429.

Canales, A. \& De los Ríos, D. (2007). Factores Explicativos de la Deserción Universitaria. Calidad de la Educación, 26, pp. 173-201.

Catterall, James S. (1998) Risk and Resilience in Student Transitions to High School. American Journal of Education, 106(2), pp. 302-333.

CIDE (2001) III Encuesta a Actores del Sistema Educativo. Santiago de Chile: Centro de Investigación y Desarrollo de la Educación.

CIDE (2003) IV Encuesta a Actores del Sistema Educativo. Santiago de Chile, Centro de Investigación y Desarrollo de la Educación.

Connell, James Patrick; Spencer, Margaret Beale \& Aber, J. Lawrence (1994) Educational Risk and Resilience in African-American Youth: Context, Self, Action, and Outcomes in School. Child Development, 65(2), Children and Poverty, pp. 493-506.

CSE (2008) Estadísticas del CSE.

CSE (2007) Estadísticas del CSE.

Donoso, S. \& Cancino, V. (2007). Caracterización Socioeconómica de los Estudiantes de Educación Superior. Calidad en la Educación, 26, pp. 205-244.

Fullana, Judit Noell (1998) La búsqueda de factores protectores del fracaso escolar en niños en situación de riesgo mediante un estudio de casos. Revista de Investigación Educativa, 16(1), pp. 2-3.

Floyd, Caren (1996) Achieving Despite the Odds: A Study of Resilience among a Group of Africa American High School Seniors. The Journal of Negro Education, 65(2), pp. 181-189.

Gladieux, L.W. \& Swail, W. (1998) Postsecondary Education: Student Success, Not Just Access. The Forgotten Half Revisited: American Youth and Young Families, 1998-2000. Washington, DC: American Youth Policy Forum.

Goldfinch \& Hughes (2007) Skills, learning styles and success of first-year undergraduates. Active Learning in Higher Education, 8, pp. 259-273.

Hays, R. B. \& Oxley, D. (1986) Social network development and functioning during a life transition. Journal of Personality and Social Psychology, 50, pp. 305-313.

Henderson, N. \& Milstein, M. M. (2003) Resiliency in schools: Making it happen for students and educators (2nd ed.). Thousand Oaks, CA: Corwin Press. 
Himmel, E. (2002) Modelos de Análisis de la deserción estudiantil en la educación superior. Calidad en la Educación, 17, pp. 91-108.

Jarret, Robin L. (1997) Resilience among Low-Income African America Youth: An Ethnographic Perspective. Ethos, 25(2), Ethnography and Sociocultural Processes: A Symposium, pp. 218-229.

Masten, A. S. (1994) Resilience in individual development: Successful adaptation despite risk and adversity. In Wang, M. C. and Gordon, G. W. (Eds.) Educational resilience in inner-city America. Hillsdale, New Jersey: Lawrence Erlbaum Associates, Inc.

Miller, M. (1995) Sources of Resilience Outcomes. Paper presented at the International Convention of the Council for Exceptional Children. (73 ${ }^{\text {rd }}$, Indianapolis, April 5-9 1995) ERIC Document. ED 381949.

Mischel, W.; Zeiss, R. \& Zeiss, A. (1974) Internal-external control and persistence: Validation and implications of the Stanford preschool internal-external scale. Journal of Personality and Social Psychology, 29, pp. 265-278.

O'Connor, Carla (2001) Comment: Making Sense of the Complexity of Social Identity in Relation to Achievement: A Sociological Challenge in the New Millennium. Sociology of Education, 74(0), Extra Issue: Current of Thought: Sociology of Education at the Dawn of the 21st Century, pp. 159-168.

O'Connor, Carla (2002) Black Women Beating the Odds from One Generation to the Next: How the Changing Dynamics of Constraint and Opportunity Affect the Process of Educational Resilience. American Educational Research Journal, 39(4), pp. 855-903.

OECD (2000). Literacy in the Information Age. Paris: Organization for Economic Co-operation and Development.

OECD (2002). Education at Glance. OECD Indicators 2002. Paris: Organization for Economic Co-operation and Development.

Pascarella, E. T. \& Chapman, D. W. (1983) A multi-institutional path analytical validation of Tinto's model of college withdrawal. American Education Research Journal, 20(1), 87-102.

Pascarella, E. \& Terenzini, P. (1991) How college affects students: Findings and insights from twenty years of research. San Francisco: Jossey-Bass.

Psacharopoulos, G. \& Patrinos, H. A. (2002) Returns to investment in education: a further update. World Bank.

Psacharopoulos, G. \& Patrinos, H. A. (2004) Returns to investment in education: a further update. Education Economics, 12. 
82 RETENCIÓN DE ESTUDIANTES UNIVERSITARIOS VULNERABLES - Andrea Canales, Danae de los Ríos

PNUD (2005) Expansión de la Educación Superior en Chile: Hacia un Nuevo Enfoque de la Equidad y Calidad. PNUD: Temas de Desarrollo Humano Sustentable \# 10.

Reed-Victor, E. \& Stronge, J. H. (2002) Homeless students and resilience: Staff perspectives on individual and environmental factors. Journal of Children \& Poverty, 159-183.

Reese, L.; Kroesen, K. \& Gallimore R. (2000) Agency and School Performance among Urban Latino Youth. In Taylor, R. \& Wang, M. (Eds.) Resilience Across Contexts: Family, Work, Culture and Community. New Jersey: Erlbaum.

Stronge, J. H. et al. (2003) Handbook on teacher evaluation. Larchmont, NY: Eye on Education.

Thomas, Liz (2002) Student retention in higher education: the role of institutional habitus. J. Education Policy, 17(4), pp. 423-442.

Tierney, William G. (1999) Models of Minority College-Going and Retention: Cultural Integrity versus Cultural Suicide. The Journal of Negro Education, 68(1), Preparing Students for the New Millennium: Exploring Factors That Contribute to the Successful Education of African American Students, pp. 80-91.

Tierney, William G.; Jun, Alexander (Mar. - Apr. 2001) A University Helps Prepare Low Income Youths for College: Tracking School Success. The Journal of Higher Education, 72(2), Special Issue: The Social Role of Higher Education, pp. 205-225.

Tinto, V. (1993) Leaving College: Rethinking the Causes and Cures of Student Attrition. Chicago: University of Chicago Press.

Tinto, V. (1987) Leaving college: Rethinking the causes and cures of student departure. Chicago: University of Chicago Press.

Tinto, V. (1975). Dropout from Higher Education: A theoretical synthesis of recent research. Review of Educational Research, 45(1), pp. 89-125.

Uribe, D. (2004) Oferta educativa y Oferta de graduados de educación superior. En Brunner, J. J. y Meller, P. (Comp.) Oferta y Demanda de Profesionales y Técnicos en Chile. Santiago de Chile, Ril Editores.

Walker, C.; Gleaves, Al. \& Grey, J. (2006) Can students within higher education learn to be resilient, and educationally speaking, does it matter? Educational Studies, 32(3), pp. 251-264.

Warren, Simon (2005) Resilience and refusal: African-Caribbean young men's agency, school exclusions, and school-based mentoring programmes. Race Ethnicity and Education, 8(3), pp. 243-259. 
Winfield, L.F. (1996) Developing Resilience in Urban Youth. NCREL Monograph. Sitio en Internet. Disponible en http://ousd.k12.ca.us/ netday/links/Partnerships/le0win.htm

World Bank (2005) World Development Indicators 2005. Washington DC.

Recibido: 28 de mayo de 2009

Aceptación: 5 de junio de 2009 